PROCEEDINGS OF THE

AMERICAN MATHEMATICAL SOCIETY

Volume 129, Number 9, Pages 2671-2678

S 0002-9939(01)05849-X

Article electronically published on February 15, 2001

\title{
SOME COMPLEMENTS TO THE JENSEN AND CHEBYSHEV INEQUALITIES AND A PROBLEM OF W. WALTER
}

\author{
S. M. MALAMUD
}

(Communicated by Albert Baernstein II)

\begin{abstract}
Motivated by an integral inequality conjectured by W. Walter, we prove some general integral inequalities on finite intervals of the real line. In addition to supplying new proofs of Walter's conjecture, the general inequalities furnish a reverse Jensen inequality under appropriate conditions and provide generalizations of Chebyshev's integral inequality.
\end{abstract}

\section{INTRODUCTION}

Let $g(s)$ be a positive nondecreasing function on $[0, x]$ and $\alpha \geq 1$. In [3], W. Walter asked whether the inequality

$$
\alpha \int_{0}^{x}(x-s)^{\alpha-1} g(s)^{\alpha} d s \leq\left(\int_{0}^{x} g(s) d s\right)^{\alpha}
$$

always holds.

A positive solution to this problem was provided by Walter and Weckesser in 4]. Independently, another proof was proposed by Egorov [2], and was announced in his talk at the 1997 Krein conference in Odessa.

After the change of variable $s=x t$, inequality (1.1) takes the form

$$
\alpha \int_{0}^{1}(1-s)^{\alpha-1} g(s)^{\alpha} d s \leq\left(\int_{0}^{1} g(s) d s\right)^{\alpha} .
$$

Now let $q$ be positive and increasing and $h$ be positive and decreasing. Then the following inequality of Chebyshev is valid:

$$
\int_{0}^{1} q(s) h(s) d s \leq \int_{0}^{1} q(s) d s \cdot \int_{0}^{1} h(s) d s .
$$

Applying (1.2) with $h(s)=\alpha(1-s)^{\alpha-1}$ and $q(s)=g(s)^{\alpha}$, we obtain an inequality with $\int_{0}^{1} g(s)^{\alpha} d s$ in the right-hand side. Hölder's inequality implies $\int_{0}^{1} g(s)^{\alpha} d s \geq$ $\left(\int_{0}^{1} g(s) d s\right)^{\alpha}$. Thus, $\left(1.1^{\prime}\right)$ is a strengthening of the Chebyshev inequality when $h=\alpha(1-s)^{\alpha-1}$.

Received by the editors September 14, 1998 and, in revised form, January 12, 2000.

1991 Mathematics Subject Classification. Primary 26D15.

Key words and phrases. Integral inequalities on the line. 
Inequality (1.1) without the factor $\alpha$ in the left-hand side was proved by Bushell and Okrasínski 1]. Their proof is rather complicated and does not provide the complete result.

In this paper we present some generalizations of inequality (1.1), with proofs different from those of [4] and [2]. Our main result, to be stated as Theorem 3.1, is the inequality

$$
\frac{\int_{0}^{1} F(g(x)) p(x) d \Phi(x)}{\int_{0}^{1} p(x) d \Phi(x)} \leq F\left(\frac{\int_{0}^{1} g(x) p(x) d x}{\int_{0}^{1} p(x) d x}\right),
$$

where $g, p$ are positive increasing functions, $F$ is convex, and $F$ and $\Phi$ are positive increasing and satisfy some additional conditions (see Theorem 3.1). In the unweighted case $p \equiv 1$, we prove in Theorem 2.1 that (1.3) holds under weaker hypotheses.

Clearly, this inequality turns into an equality if $g=$ const. It seems reasonable that the following conjecture is valid.

Conjecture. Equality in (1.3) holds if and only if $g=$ const.

On the one hand, (1.3) may be considered as a converse to Jensen's inequality. On the other hand, when Theorem 2.1 is applied with $F(x)=x$ and $\Phi$ concave, we recover Chebysev's inequality. Inequality $\left(1.1^{\prime}\right)$ is the special case of $(1.3)$ when $F(x)=x^{\alpha}, \Phi(x)=1-(1-x)^{\alpha}$, and $p \equiv 1$. Thus, $\left(1.1^{\prime}\right)$ is a consequence of both Theorem 2.1 and Theorem 3.1.

The initial version of this paper, which contained a proof of (1.1), was submitted for publication before I knew about the paper [4]. Later, I received a letter from Professor Walter in which he enclosed a copy of [4].

\section{The UnWeighted Inequality}

2.1. Here we present our first converse to Jensen's inequality. Let $C$ be a real number, $F$ a differentiable function on $(0, \infty)$, and $\Phi$ a function on $[0,1]$. Our results will make use of the following condition involving $C, F$ and $\Phi$ :

$$
F^{\prime}(\lambda) \frac{\Phi(1)-\Phi(x)}{1-x} \leq C F^{\prime}(\lambda(1-x)), \quad 0<x<1, \quad 0<\lambda<\infty .
$$

Theorem 2.1. Let $g$ be a positive increasing function on $[0,1], \Phi$ a positive function of bounded variation on $[0,1]$, and $F:(0, \infty) \rightarrow \mathbb{R}$ positive, increasing, convex and differentiable. If (2.1) is satisfied with $C=\Phi(1)-\Phi(0)>0$, then the inequality

$$
\frac{\int_{0}^{1} F(g(x)) d \Phi(x)}{\int_{0}^{1} d \Phi(x)} \leq F\left(\int_{0}^{1} g(x) d x\right)
$$

is valid.

Proof. i) Let $g(x)$ be a positive increasing step function:

$$
g(x)=\left\{g_{i}: x \in((i-1) / n, i / n]\right\}, 0 \leq g_{1} \leq g_{2} \leq \ldots \leq g_{n} .
$$


Assume also, without loss of generality, that $\Phi(1)-\Phi(0)=1$. The required inequality takes the form

$$
\sum_{i=1}^{n} F\left(g_{i}\right)\left(\Phi\left(\frac{i}{n}\right)-\Phi\left(\frac{i-1}{n}\right)\right) \leq F\left(\frac{g_{1}+\ldots+g_{n}}{n}\right) .
$$

Let $\lambda:=g_{n} \in\left[g_{n-1}, \infty\right)$. Denote by $r(\lambda)$ the difference of the right-hand side and the left-hand side of (2.3). Then, since $F$ is convex, one obtains, using (2.1),

$$
\begin{aligned}
r^{\prime}(\lambda)=\frac{1}{n} F^{\prime}\left(\frac{g_{1}+\ldots+\lambda}{n}\right) & -F^{\prime}(\lambda)\left(\Phi(1)-\Phi\left(\frac{n-1}{n}\right)\right) \\
& \geq \frac{1}{n} F^{\prime}\left(\frac{\lambda}{n}\right)-F^{\prime}(\lambda)\left(\Phi(1)-\Phi\left(\frac{n-1}{n}\right)\right) \geq 0 .
\end{aligned}
$$

Thus $r(\lambda)$ increases and it suffices to prove $(2.3)$ for $g_{n-1}=g_{n}$ (since $\left.g_{n-1} \leq g_{n}\right)$. In the same way, setting $\lambda=g_{n-1}$ we reduce the inequality to the case $g_{n-1}=g_{n-2}$. Continuing the process we arrive at the obvious equality $\sum_{1}^{n} \Phi((i-1) / n)-\Phi(i / n)=$ 1.

ii) To complete the proof it suffices to observe that an arbitrary monotone increasing function may be approximated by step functions and then pass to the limit in $(2.3)$.

By an approximation argument, the assumption in Theorem 2.1 that $F$ be differentiable can be omitted.

For $\Phi(x)=1-(1-x)^{\alpha}, F(x)=x^{\alpha}$ and $C=1,(2.1)$ holds with equality. The resulting inequality $(2.2)$ then becomes $\left(1.1^{\prime}\right)$, the inequality conjectured by Walter. For $1 \leq \beta \leq \alpha$, the pair $\Phi(x)=1-(1-x)^{\alpha}$ and $F(x)=x^{\beta}$ also satisfies the hypotheses of Theorem 2.1 with $C=1$. This provides a generalization of $\left(1.1^{\prime}\right)$ :

$$
\alpha \int_{0}^{1}(1-s)^{\alpha-1} g(s)^{\beta} d s \leq\left(\int_{0}^{1} g(s) d s\right)^{\beta}, \quad 1 \leq \beta \leq \alpha .
$$

This generalization was also proved by Walter and Weckesser in [4]. For $\beta=1$ the inequality is a special case of Chebyshev's inequality, while for $\beta \in(0,1)$ it follows from Hölder's inequality and the case $\beta=1$.

If we take $F(x)=x$, then condition (2.1) with $C=\Phi(1)-\Phi(0)$ can be written as

$$
(1-x) \Phi(0)+x \Phi(1) \leq \Phi(x), \quad x \in[0,1] .
$$

Writing $g=q$, Theorem 2.1 implies

Corollary 2.1. Let $q$ be positive and increasing on $[0,1]$ and let $\Phi$ be positive and of bounded variation on $[0,1]$ with $\Phi(1)-\Phi(0)>0$. If $\Phi$ satisfies $\left(2.1^{\prime}\right)$, then

$$
\int_{0}^{1} q(x) d \Phi(x) \leq \int_{0}^{1} q(x) d x \int_{0}^{1} d \Phi(x)
$$

If $h$ is positive and decreasing on [0,1], then $\Phi(x)=\int_{0}^{x} h(s) d s$ is concave, and hence satisfies $\left(2.1^{\prime}\right)$. Thus, $\left(1.2^{\prime}\right)$ provides a strengthening of the Chebyshev inequality (1.2). 
2.2. Condition (2.1) is sometimes difficult to check. Therefore it is worthwhile to seek conditions sufficient for the validity of (2.1). The following lemma provides such conditions. To state it we recall that a function $\varphi(x)$ positive on $[0,1]$ is called logarithmically concave if the function $\ln \varphi\left(e^{x}\right)$ is concave on $(-\infty, 0)$.

Lemma 2.1. Let $F$ be an increasing twice differentiable function on $(0, \infty)$, and $\Phi$ a differentiable function on $[0,1]$ with $\Phi(1)>\Phi(x)$ for all $x \in[0,1)$ and $\Phi(1)-$ $\Phi(0)=1$. Set

$$
f(\lambda)=\ln F^{\prime}\left(e^{\lambda}\right), \quad \lambda \in \mathbb{R},
$$

and

$$
\varphi(x)=\Phi(1)-\Phi(1-x), \quad x \in(0,1), \quad \psi(x)=\ln \varphi\left(e^{x}\right), \quad x \in(-\infty, 0) .
$$

1) If

$$
f^{\prime}(\lambda) \leq \min _{[-\infty, 0]} \psi^{\prime}(x)-1:=a-1 \quad \forall \lambda \in \mathbb{R},
$$

then, for $0<x<1$,

$$
F^{\prime}(\lambda(1-x)) \geq F^{\prime}(\lambda)(1-x)^{a-1} \geq F^{\prime}(\lambda) \frac{\Phi(1)-\Phi(x)}{1-x}
$$

and thus (2.1) holds with $C=1$.

2) If $\varphi$ is logarithmically concave, then (2.1) is equivalent to (2.4). In this case $a=\Phi^{\prime}(0)$ and (2.4) takes the form $f^{\prime}(\lambda) \leq \Phi^{\prime}(0)-1$.

Proof. 1) The first inequality of (2.5) follows from $f^{\prime} \leq a-1$ and the second from $\psi^{\prime} \geq a$, together with applications of Lagrange's mean value theorem.

2) Now let $\varphi$ be logarithmically concave. Then $\psi^{\prime}(x)$ attains its minimum on the negative semi-axis at the point $x=0$. Since $\psi^{\prime}(x)=\varphi^{\prime}\left(e^{x}\right) e^{x} / \varphi\left(e^{x}\right)$, then $\min \psi^{\prime}(x)=\psi^{\prime}(0)=\varphi^{\prime}(1)=\Phi^{\prime}(0)$. Conversely, letting $x$ tend to 0 , one obtains $f^{\prime}(\lambda) \leq \psi^{\prime}(0)-1$.

Combining Lemma 2.1 and Theorem 2.1, we obtain the following corollary.

Corollary 2.2. Let $g$ be a positive increasing function on $[0,1], \Phi$ a positive differentiable function of bounded variation on $[0,1]$, with $\Phi(1)>\Phi(x)$ for all $x \in[0,1)$ and $\Phi(1)-\Phi(0)=1$. Let $F:(0, \infty) \rightarrow \mathbb{R}$ be positive, increasing, convex and twice differentiable, and let $f$ and $\psi$ be defined as in Lemma 2.1 .

1) If $0 \leq f^{\prime}(\lambda) \leq \min _{[-\infty, 0]} \psi^{\prime}(x)-1$, then (2.2) holds.

2) If $\varphi$ is logarithmically concave and $f^{\prime}(\lambda) \leq \Phi^{\prime}(0)-1$, then (2.2) holds.

\section{The Weighted inequality}

Here we establish a weighted analogue of Theorem 2.1.

Theorem 3.1. Let $\Phi, p$ and $g$ be positive and increasing on $[0,1]$, and let $F$ be positive, increasing and convex on $(0, \infty)$. Assume also that $\Phi$ is concave and that $F$ and $\Phi$ are such that (2.4) is satisfied. Then the inequality

$$
\frac{\int_{0}^{1} F(g(x)) p(x) d \Phi(x)}{\int_{0}^{1} p(x) d \Phi(x)} \leq F\left(\frac{\int_{0}^{1} g(x) p(x) d x}{\int_{0}^{1} p(x) d x}\right)
$$

is valid. 
The difference between Theorems 3.1 and 2.1 is the presence of the weight $p(x)$. To pay for the increased generality, we imposed in Theorem 3.1 the additional hypotheses that $\Phi$ is concave, and changed (2.1) to the stronger condition (2.4).

To prove the theorem, we need some lemmas.

Lemma 3.1. Let $\Phi(x)$ be as in Theorem 3.1, and let $\left\{p_{k}\right\}_{1}^{n}$ be a nondecreasing positive sequence. Then the inequality

$$
\sum_{k=1}^{n}\left(\Phi\left(\frac{k}{n}\right)-\Phi\left(\frac{k-1}{n}\right)\right) p_{k} \leq(\Phi(1)-\Phi(0)) \cdot \frac{p_{1}+\ldots+p_{n}}{n}
$$

is valid.

Proof. Such a $\Phi$ satisfies the hypotheses of Corollary 2.1. To obtain Lemma 3.1, apply Corollary 2.1 with $q$ the appropriate step function.

Lemma 3.2. Let $0 \leq p_{1} \leq p_{2} \leq \ldots \leq p_{n}$. Then

$$
\frac{p_{n}+p_{n-1}+\ldots+p_{n-k+1}}{p_{n}+p_{n-1}+\ldots+p_{1}} \geq \frac{k}{n}, \quad 1 \leq k \leq n .
$$

Proof. Let $s_{1}:=p_{n}+\ldots+p_{n-k+1}, s_{2}:=p_{1}+\ldots+p_{n-k}$. Then obviously $s_{1} \geq$ $k p_{n-k+1}$ and $s_{2} \leq(n-k) p_{n-k+1}$. Further, the function $s_{1} /\left(s_{1}+s_{2}\right)=1-s_{2} /\left(s_{1}+s_{2}\right)$ increases in $s_{1}$ and decreases in $s_{2}$. Therefore

$$
\frac{s_{1}}{s_{1}+s_{2}} \geq \frac{k p_{n-k+1}}{k p_{n-k+1}+(n-k) p_{n-k+1}}=\frac{k}{n}
$$

which is the desired inequality.

Lemma 3.3. Let $\Phi$ and $\psi$ be as in Theorem 3.1, $a=\min \psi^{\prime}(x)$ and let $\left\{p_{i}\right\}_{1}^{n}$ be a positive nondecreasing sequence. Assume also that $\Phi(1)-\Phi(0)=1$. Then for each $k \in\{1, \ldots, n\}$ we have

$$
\begin{gathered}
\left(\frac{p_{n-k+1}+\ldots+p_{n}}{p_{1}+\ldots+p_{n}}\right)^{a} \cdot\left[\sum_{i=1}^{n}\left(\Phi\left(\frac{i}{n}\right)-\Phi\left(\frac{i-1}{n}\right)\right) p_{i}\right] \\
\geq \sum_{i=n-k+1}^{n}\left(\Phi\left(\frac{i}{n}\right)-\Phi\left(\frac{i-1}{n}\right)\right) p_{i} .
\end{gathered}
$$

Proof. Let $\varphi(x)=\Phi(1)-\Phi(1-x)$. Rewrite (3.4) in the form

$$
\begin{aligned}
& {\left[\sum_{i=1}^{n}\left(\varphi\left(\frac{n-i+1}{n}\right)-\varphi\left(\frac{n-i}{n}\right)\right) p_{i}\right]} \\
& \quad \geq\left(\frac{p_{1}+\ldots+p_{n}}{p_{n-k+1}+\ldots+p_{n}}\right)^{a} \cdot \sum_{i=n-k+1}^{n}\left(\varphi\left(\frac{n-i+1}{n}\right)-\varphi\left(\frac{n-i}{n}\right)\right) p_{i} .
\end{aligned}
$$

The right-hand side of (3.5) is a convex function of $p_{1}$ for $0 \leq p_{1} \leq p_{2}$, and the left-hand side is a linear function. Therefore it suffices to prove $(3.5)$ for $p_{1}=0$ and for $p_{1}=p_{2}$. Thus we have eliminated $p_{1}$. In the same way we can eliminate $p_{2}$ 
etc. Continuing the process we arrive at

$$
\begin{aligned}
& {\left[\left(\varphi\left(\frac{m}{n}\right)-\varphi\left(\frac{k}{n}\right)\right) p_{n-k+1}+\sum_{i=n-k+1}^{n}\left(\varphi\left(\frac{n-i+1}{n}\right)-\varphi\left(\frac{n-i}{n}\right)\right) p_{i}\right]} \\
& \geq\left(\frac{(m-k) p_{n-k+1}+p_{n-k+1}+\ldots+p_{n}}{p_{n-k+1}+\ldots+p_{n}}\right)^{a} \\
& \quad \times \sum_{i=n-k+1}^{n}\left(\varphi\left(\frac{n-i+1}{n}\right)-\varphi\left(\frac{n-i}{n}\right)\right) p_{i} .
\end{aligned}
$$

with some $m, k \leq m \leq n$. Write

$$
\begin{aligned}
& \lambda_{1}=p_{n-k+1}, \\
& \lambda_{2}=\sum_{n-k+1}^{n}[\varphi((n-i+1) / n)-\varphi((n-i) / n)] p_{i} \\
& \lambda_{3}=p_{n-k+1}+\ldots+p_{n} .
\end{aligned}
$$

Making use of Lemma 3.1 one obtains

$$
0 \leq \varphi\left(\frac{k}{n}\right) \lambda_{1} \leq \lambda_{2} \leq \frac{1}{k} \varphi\left(\frac{k}{n}\right) \cdot \lambda_{3},
$$

and (3.6) takes the form

$$
\left[\left(\varphi\left(\frac{m}{n}\right)-\varphi\left(\frac{k}{n}\right)\right) \lambda_{1}+\lambda_{2}\right] \geq\left(\frac{(m-k) \lambda_{1}+\lambda_{3}}{\lambda_{3}}\right)^{a} \cdot \lambda_{2} .
$$

The right-hand side of (3.7) is convex in $\lambda_{1}$ and the left-hand side is linear. Hence, it suffices to check the inequality for $\lambda_{1}=0$ and for $\lambda_{2}=\varphi(k / n) \lambda_{1}$. The first case is trivial. Let $\lambda_{2}=\varphi(k / n) \lambda_{1}$. Then (3.7) takes the form

$$
\varphi\left(\frac{m}{n}\right) \lambda_{1} \geq\left(\frac{(m-k) \lambda_{1}+\lambda_{3}}{\lambda_{3}}\right)^{a} \cdot \varphi\left(\frac{k}{n}\right) \lambda_{1} .
$$

But $\lambda_{3} \geq k \lambda_{1}$ and hence

$$
\left(\frac{(m-k) \lambda_{1}+\lambda_{3}}{\lambda_{3}}\right)^{a}=\left(\frac{(m-k) \lambda_{1}}{\lambda_{3}}+1\right)^{a} \leq\left(\frac{m}{k}\right)^{a} .
$$

Therefore it suffices to prove the inequality $\varphi(x y) \geq x^{a} \varphi(y), \quad$ for $x \in[1,1 / y], y \in$ $(0,1)$. After simple transformations this inequality takes the form

$$
\frac{\psi(x+y)-\psi(y)}{x} \geq a .
$$

This inequality follows from the definition of $a$.

Now we are ready to prove Theorem 3.1.

Proof. As in the proof of Theorem 2.1, the proof can be reduced to the case when $g(x)$ is a positive increasing step function:

$$
g(x)=\left\{g_{i}: x \in((i-1) / n, i / n]\right\}, 0 \leq g_{1} \leq g_{2} \leq \ldots \leq g_{n} .
$$


Assume that $\Phi(1)-\Phi(0)=1$. The required inequality takes the form

$$
\begin{gathered}
F\left(\frac{g_{1} p_{1}+\ldots+g_{n} p_{n}}{p_{1}+\ldots+p_{n}}\right) \sum_{i=1}^{n}\left[\Phi\left(\frac{i}{n}\right)-\Phi\left(\frac{i-1}{n}\right)\right] p_{i} \\
\geq \sum_{i=1}^{n}\left[\Phi\left(\frac{i}{n}\right)-\Phi\left(\frac{i-1}{n}\right)\right] F\left(g_{i}\right) p_{i} .
\end{gathered}
$$

Now let $\lambda=g_{n} p_{n}$ (here $p_{n}$ is fixed and $g_{n}$ will vary) and let $r(\lambda)$ be the difference between the left-hand side and the right-hand side of (3.9). Since $F^{\prime}(x)$ increases, we have

$$
F^{\prime}\left(\frac{g_{1} p_{1}+\ldots+g_{n-1} p_{n-1}+\lambda}{p_{1}+\ldots+p_{n}}\right) \geq F^{\prime}\left(\frac{\lambda}{p_{1}+\ldots+p_{n}}\right)
$$

and therefore

$$
\begin{aligned}
r^{\prime}(\lambda) \geq & \frac{1}{\sum_{1}^{n} p_{i}} F^{\prime}\left(\frac{\lambda}{p_{1}+\ldots+p_{n}}\right) \sum_{i=1}^{n}\left[\Phi\left(\frac{i}{n}\right)-\Phi\left(\frac{i-1}{n}\right)\right] p_{i} \\
& -\left[\Phi(1)-\Phi\left(\frac{n-1}{n}\right)\right] F^{\prime}\left(\frac{\lambda}{p_{n}}\right) .
\end{aligned}
$$

From the hypothesis of the theorem and Lemma 2.1 we deduce

$$
F^{\prime}\left(\frac{\lambda}{p_{1}+\ldots+p_{n}}\right)=F^{\prime}\left(\frac{p_{n}}{p_{1}+\ldots+p_{n}} \cdot \frac{\lambda}{p_{n}}\right) \geq\left(\frac{p_{n}}{\sum_{1}^{n} p_{i}}\right)^{a-1} \cdot F^{\prime}\left(\frac{\lambda}{p_{n}}\right) .
$$

Thus to prove that $r^{\prime}(\lambda) \geq 0$ it suffices to establish the inequality

$$
\left(\frac{p_{n}}{p_{1}+\ldots+p_{n}}\right)^{a} \cdot\left[\sum_{i=1}^{n}\left(\Phi\left(\frac{i}{n}\right)-\Phi\left(\frac{i-1}{n}\right)\right) p_{i}\right] \geq\left(\Phi(1)-\Phi\left(\frac{n-1}{n}\right)\right) p_{n} .
$$

Observe that (3.10) coincides with (3.4) for $k=1$. Thus, by virtue of Lemma $3.3, r(\lambda)$ increases and hence it suffices to prove (3.9) in the case $g_{n-1}=g_{n}$ :

$$
\begin{gathered}
F\left(\frac{\sum_{1}^{n-2} g_{i} p_{i}+\left(p_{n-1}+p_{n}\right) g_{n-1}}{p_{1}+\ldots+p_{n}}\right) \sum_{i=1}^{n}\left[\Phi\left(\frac{i}{n}\right)-\Phi\left(\frac{i-1}{n}\right)\right] p_{i} \\
\geq \sum_{i=1}^{n-2}\left[\Phi\left(\frac{i}{n}\right)-\Phi\left(\frac{i-1}{n}\right)\right] F\left(g_{i}\right) p_{i} \\
\quad+F\left(g_{n-1}\right) \sum_{i=n-1}^{n}\left[\Phi\left(\frac{i}{n}\right)-\Phi\left(\frac{i-1}{n}\right)\right] p_{i} .
\end{gathered}
$$

In the same way, setting $\lambda=g_{n-1}\left(p_{n-1}+p_{n}\right)$ and making use of Lemma 3.3 for $k=2$, one reduces (3.11) to the case $g_{n-1}=g_{n-2}$. Continuing this process, we arrive at an equality. Theorem 3.1 is proved. 
If in Theorem 3.1 we take $F(\lambda)=\lambda^{\beta}$ and apply Lemma 2.12 ), we obtain

Corollary 3.2. Let $\Phi, p, g$ and $\phi$ be as in Theorem 3.1, and suppose that $\phi$ is logarithmically concave. Then

$$
\frac{\int_{0}^{1}(g(x))^{\beta} p(x) d \Phi(x)}{\int_{0}^{1} p(x) d \Phi(x)} \leq\left(\frac{\int_{0}^{1} g(x) p(x) d x}{\int_{0}^{1} p(x) d x}\right)^{\beta}, \quad 1 \leq \beta \leq a:=\frac{\Phi^{\prime}(0)}{\Phi(1)-\Phi(0)} .
$$

The choice $p \equiv 1, \Phi(x)=1-(1-x)^{\alpha}$ proves again the " $\beta$ generalization" of Walter's conjecture $\left(1.1^{\prime}\right)$ which we already proved in $\S 2$.

\section{ACKNOWLEDGEMENTS}

I am deeply indebted to Professor Yu. V. Egorov for a prepublication view of his work 2] and to Professor W. Walter for informing me about 4].

I also express my deep gratitude to Professor A. Baernstein for very useful remarks and comments, which improved the paper.

\section{ADDED AFTER POSTING}

While this paper was in publication the author was informed about the paper "Weighted inequalities for monotone and concave functions" by H. Heinig and L. Maligranda (Studia Math. 116 (1995), 133-165), where they also generalize inequality (1.1) and use it to obtain weighted Muckenhoupt-type results for arbitrary and monotone functions.

\section{REFERENCES}

1. P.J. Bushell and W. Okrasínski, Nonlinear Volterra Integral Equations with Convolution Kernel, J. London Math. Soc. (2)41 (1990), 503-510. MR 91g:45001

2. Yu. V. Egorov, On the best constant in a Poincaré-Sobolev inequality, Operator Theory: Advances and Applications (to appear).

3. W. Walter, Problem: An integral inequality by Bushell and Okrasinski, Intern. series of Numerical Mathematics Vol. 103 (1992).

4. W. Walter and V. Weckesser, An integral inequality of convolution type, Aequationes Mathematicae 46 (1993), 369-376. MR 94e:26030

Department of Mathematics, Donetsk State University, Universitetskaya Str. 24, DONETSK 340055, UKRAINe

E-mail address: MMM@univ.donetsk.ua 